\title{
Comparing the effects of different dietary organic acids on the growth, intestinal short- chain fatty acids, and liver histopathology of red hybrid tilapia (Oreochromis sp.) and potential use of these as preservatives
}

\begin{abstract}
Dietary organic acids are increasingly being investigated as a potential means of improving growth and nutrient utilization in aquatic animals. A 9-week study was performed to compare equal amounts $(2 \%)$ of different organic acids (sodium butyrate, acetate, propionate, or formate) on the growth, muscle proximate composition, fatty acid composition, cholesterol and lipid peroxidation, differential cell counts, plasma biochemistry, intestinal short-chain fatty acid (SCFA) level, and liver histopathology to red hybrid tilapia (Oreochromis sp.) (initial mean weight of $2.87 \mathrm{~g}$ ). A second experiment was performed to determine their effects on lipid peroxidation and trimethylamine (TMA) when added at $1 \%$ to tilapia meat and left out for $24 \mathrm{~h}$. The results of the first experiment showed no treatment effect to growth, feeding efficiencies, or muscle fatty acid composition, but all dietary organic acids significantly decreased intestinal SCFA. Dietary butyrate and propionate significantly decreased muscle lipid peroxidation compared to the control group, but the dietary formate treatment had the lowest lipid peroxidation compared to all treatments. Muscle crude protein and lipid in tilapia fed the formate diet were significantly lower and higher, respectively, and showed evidence of stress based on the differential cell counts, significantly higher plasma glucose and liver glycogen, as well as inflammatory responses in the liver. Although a potential benefit of dietary organic acids was a reduction to lipid peroxidation, this could be accomplished post-harvest by direct additions to the meat. In addition, inclusions of butyrate and propionate to tilapia meat significantly decreased TMA, which might be a more costeffective option to improve the shelf life of tilapia products.
\end{abstract}

Keyword: Glycogen; Inflammation; Lipid peroxidation; Liver histology; Organic acids; Short-chain fatty acids; Trimethylamine 